
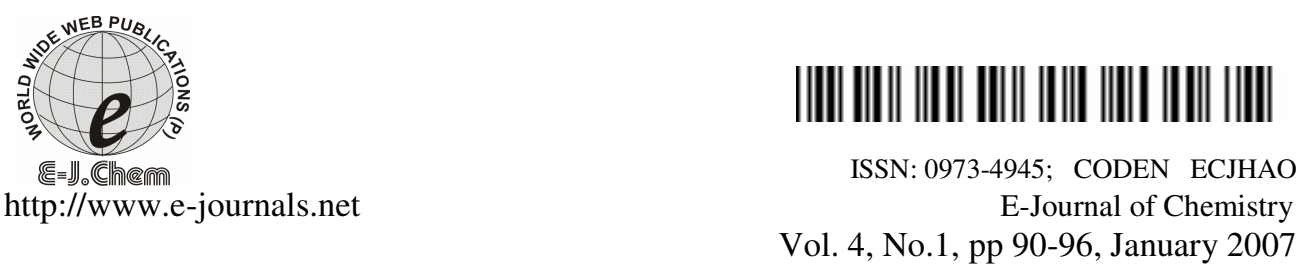

\title{
Synthesis of Some Aryl Chalcones Using Silica- Sulphuric Acid Reagent under Solvent Free Conditions
}

\author{
G.THIRUNARAYANAN* and G. VANANGAMUDI \\ *Department of Chemistry, \\ Annamalai University, Annamalainagar-608 002, India. \\ Department of Chemistry, \\ Government Arts College, Chidambaram-608 102, India. \\ drgtnarayanan@gmail.com
}

Received 29 September 2006; Accepted 1 November 2006

\begin{abstract}
There are two series of $\alpha, \beta$-unsaturated ketones derived forn Biphenyl and 9H-Fluorenyl, and ketones with various substituter benzaldehydes under solvent free conditions using silica-sulphuric acid as a: reagent in an oven. The catalyst silica is reusable and the yields of chalcone: are more than $90 \%$. These chalcones are characterized by physical constant: and spectral data.
\end{abstract}

Keywords: Chalcones, solvent free synthesis, infrared spectra, NMR spectra, environmental begin reaction

\section{Introduction}

The adlol reaction is an important carbon-carbon bond formation reaction in organic chemistry ${ }^{1}$ involving the addition of an enol or enolate anion to an aldehydes or ketones ${ }^{2}$. In this aldol addition, the reaction results in a $\beta$-hydroxy ketones or aldehydes also called an "aldol" (aldehydes + alcohol). The initial aldol adduct undergoes dehydration (loss of water) to form an $\alpha, \beta$-unsaturated ketones or aldehydes. The $\alpha, \beta$-unsaturated ketones are chalcones and they have great existence in the plat kingdom. It is well-known that most of the natural or synthetic chalcones are highly biologically active with a great pharmaceuticals and medicinal applications ${ }^{3}$. Recently they are used as anti-AIDS ${ }^{4}$ agents, cytotoxic with antiangiogenic activity ${ }^{5}$, antimalarial ${ }^{6}$, anti-inflammatory ${ }^{7}$ and antitumor ${ }^{8}$. 
The U.S. Environmental Protection Agency (EPA) has suggested a drastic reduction of using of more than ten hazard of common organic solvents in the industrial production of chemicals. The authors dealing in this paper a cleaner and safe production of high yield of stereoselective aryl chalcones as an important biologically active compound in silica-sulfuric acid as a reagent and environmental friendly reaction medium. There has been tremendous interest in the application of solvent free aldol ${ }^{9}$ and crossed-aldol $^{10}$ reactions which are employed for synthesis of carbonyl compounds due to the operational simplicity, easier work-up, better yield and eco-friendly nature.. The condensation of ketones with aldehydes is of special interest and the crossed-aldol condensation is an effective pathway for those compounds preparations. However the traditional acid-base catalyzed reactions suffer from the reverse reaction ${ }^{11}$ and self condensation of starting molecules ${ }^{12}$.

Various reagents and Co-ordination complexes of $\mathrm{Mn}$ (II), $\mathrm{Fe}$ (II), $\mathrm{Co}$ (II), $\mathrm{Ni}$ (II), $\mathrm{Cu}$ (II) and $\mathrm{Zn}$ (II) ions with various ligands have been employed for aldol condensation ${ }^{13}$. Metal salts of $\mathrm{Cp}_{2} \mathrm{ZrH}_{2}$ are used for condensation of cycloalkanones ${ }^{14} . \mathrm{KF}-\mathrm{Al}_{2} \mathrm{O}_{3}$ and bis ( $p$-methoxy phenyl) tellurides are have been used for crossed condensation under microwave irradiation ${ }^{15}$. Anhydrous $\mathrm{RuCl}_{3}$ and $\mathrm{TiCl}_{3}\left(\mathrm{SO}_{3} \mathrm{CF}_{3}\right)$ have also been applied for aldol condensation reactions under solvent free conditions ${ }^{16}$. Now more attention has been paid to synthesis of acyclic and cyclic chalcones by chemists and scientists ${ }^{17}$. Balakrishna Kalluraya ${ }^{18}$ et.al. reported to obtain $60-$ $70 \%$ yield of sydnone chalcones under solvent free condition by aldol condensation reaction by grinding of ketones and aldehydes with sodium hydorxide. D.D.Sung et. al ${ }^{19}$ derived some biphenyl and fluorenyl chalcones by base catalyzed crossed aldol reaction, but they do not discussed the yield of product. Hassan et. $\mathrm{al}^{20}$. Synthesized $62-95 \%$ yields of some hetero aryl chalcones by condensation of heterorylketones and aldehydes with $\mathrm{NaOH}$ in water under cooling condition of aldol reaction. Along this line the author have introduced potentially useful reagent for stereoselective crossed-aldol deprotection of carbonyl groups. Therefore the author decides to choice of a new reagent of reagent system to overcome the limitations of reactions. In addition, for our purpose clean, clear and easy workup was also important. On the other hand any condensation in the amount of sulfuric acid needed and or any simplification in handling procedures is require for risky, economic advantage and environment potection ${ }^{21}$. In addition there is current research and general interest in heterogeneous system because of the importance of such systems has in industry and in developing technologies ${ }^{22}$. In continuation of earlier work $^{23}$ on the application of inorganic acid salts ${ }^{24}$, the author have prepared that silica gel reacts with chloro sulfonic acid to give silica-sulfuric acid $\left[\mathrm{SiO}_{2}-\mathrm{OSO}_{3} \mathrm{H}\right]$. It is interesting to note that the reaction is easy and clean without any workup procedure, because $\mathrm{HCl}$ gas is evolved from the reaction vessel immediately, therefore the author finds that silica-sulfuric acid is an excellent reagent for acid sulfuric replacement in organic reactions without any limitations like condensation of activated aromatic ring with sensitive functional groups. Since the heterogeneous reagent system have many advantages such as simple experimental procedure, mild conditions and to minimization of chemical wastes as compared to their liquid phase counterparts $^{25}$. So silica-sulfuric acid is used as a versatile and stable solid catalyst for crossedaldol reactions due to it gave more than $90 \%$ of products.. In this article the author would like to report an efficient and selective method for condensation of Biphenyl and 9H-Fluorenyl ketones with various substituted benzaldehydes under solvent free conditions using silica-sulphuric acid as a reagent in an oven to yields the respective E-2-propen-1-ones. The promoting effect of silica - sulphuric acid in their reaction was shown good performance and it is proved by obtaining higher percentage of yields. The product was isolated and the remaining catalyst was washed and reused with fresh substrate for further reactions. No decrease in the yield 
was observed, demonstrating that silica-sulphuric acid can be reused in crossed-aldol condensation reaction without environmental discharge.

\section{Experimental}

\section{General Procedures}

All chemicals and Analytical Grade solvents were purchased from E-Merck chemical company. Melting points of all chalcones were determined in open glass capillaries on Mettler FP51 melting point apparatus and are uncorrected. Infrared spectra $(\mathrm{KBr}, 4000-$ $400 \mathrm{~cm}-1)$ were recorded on a Perkin-Elmer Fourier transform spectrophotometer. The nuclear magnetic resonance spectra, both ${ }^{1} \mathrm{H}$ - and ${ }^{13} \mathrm{C}$-, of chalcones were recorded using UNITYPLUS-300 "KIBSIPS" $300 \mathrm{MHz}$ spectrometer. Micro analyses of chalcones were performed using a Perkin-Elmer 240C Analyzer.

General procedure for crossed-aldol condensation of aromatic ketones with benzaldehydes

Biphenyl and $9 \mathrm{H}$-fluorenyl ketones $(2 \mathrm{mmol}), m$-and $p$-substituted benzaldehydes $(4.2 \mathrm{~mol})$ and silica-sulfuric acid( $1.5 \mathrm{~g}$ equal to $4 \mathrm{mmol}$ of $\left.\mathrm{H}^{+}\right)$were mixed thoroughly, placed in a glass tube and capped (Scheme 1). The mixture was heated in an oven at $80^{\circ} \mathrm{C}$ for $2-3.5 \mathrm{~h}$. After complete conversion of the ketones as monitored by TLC, the mixture was cooled to room temperature. Dichloromethane $(20-30 \mathrm{ml})$ was added and heated for 3-5 minutes. The reagent was removed by filtration. The filtrate was concentrated and the solid residue was recrystallised from ethanol to afford the pure products as pale yellow glittering solid. The catalyst was recycled by washing the solid reagent remained on the filter by ethyl acetate ( 20 $\mathrm{ml}$ ) followed by drying in an oven at $50^{\circ} \mathrm{C}$ for $2 \mathrm{~h}$. and can be reusable for another reaction run. They are characterized by comparison of their physical constants (Table-1) and spectral data reported in literature. Spectral and microanalysis data of selective compounds are summarized below.

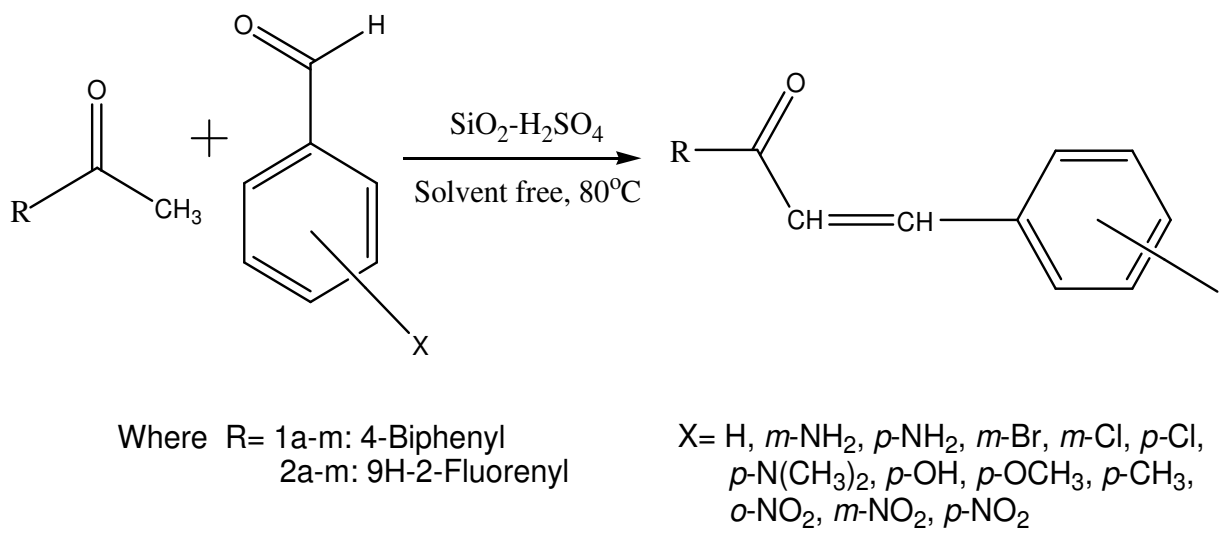

\section{Scheme 1}


Table 1. Physical constants and the yields of chalcones 1a-2m.

\begin{tabular}{|c|c|c|c|c|c|}
\hline $\begin{array}{c}\text { Compd. } \\
\text { No. }\end{array}$ & $\mathrm{R}$ & $X$ & $\begin{array}{l}\text { Molecular } \\
\text { Formula } \\
\end{array}$ & $\begin{array}{l}\text { Yield } \\
\% \\
\end{array}$ & $\begin{array}{l}\text { M.P } \\
{ }^{\circ} \mathrm{C} \\
\end{array}$ \\
\hline $1 \mathrm{a}$ & 4-Biphenyl & $\mathrm{H}$ & $\mathrm{C}_{21} \mathrm{H}_{16} \mathrm{O}$ & 91 & $155-156\left(156^{19 b}\right)$ \\
\hline $1 b$ & , & $m-\mathrm{NH}_{2}$ & $\mathrm{C}_{21} \mathrm{H}_{17} \mathrm{NO}$ & 92 & $115-116$ \\
\hline $1 \mathrm{c}$ & , & $p-\mathrm{NH}_{2}$ & $\mathrm{C}_{21} \mathrm{H}_{17} \mathrm{NO}$ & 94 & $85-86$ \\
\hline $1 d$ & ", & $m-\mathrm{Br}$ & $\mathrm{C}_{21} \mathrm{H}_{15} \mathrm{BrO}$ & 95 & $117-118$ \\
\hline $1 \mathrm{e}$ & , & $m-\mathrm{Cl}$ & $\mathrm{C}_{21} \mathrm{H}_{15} \mathrm{ClO}$ & 95 & $153-154\left(152-153^{19 b}\right)$ \\
\hline 1f & $"$ & $p-\mathrm{Cl}$ & $\mathrm{C}_{21} \mathrm{H}_{15} \mathrm{ClO}$ & 95 & $184-185\left(183-184^{19 b}\right)$ \\
\hline $1 \mathrm{~g}$ & $"$ & $p-\mathrm{N}\left(\mathrm{CH}_{3}\right)_{2}$ & $\mathrm{C}_{23} \mathrm{H}_{21} \mathrm{NO}$ & 94 & $118-119$ \\
\hline $1 \mathrm{~h}$ & , & $p-\mathrm{OH}$ & $\mathrm{C}_{21} \mathrm{H}_{16} \mathrm{O}_{2}$ & 94 & $110-111\left(109-110^{19 b}\right)$ \\
\hline $1 \mathrm{i}$ & $"$ & $p-\mathrm{OCH}_{3}$ & $\mathrm{C}_{22} \mathrm{H}_{18} \mathrm{O}_{2}$ & 92 & $122-123\left(123-124^{19 b}\right)$ \\
\hline $1 \mathrm{j}$ & " & $p-\mathrm{CH}_{3}$ & $\mathrm{C}_{22} \mathrm{H}_{18} \mathrm{O}$ & 94 & $116-117\left(117-118^{19 b}\right)$ \\
\hline $1 \mathrm{k}$ & , & $o-\mathrm{NO}_{2}$ & $\mathrm{C}_{21} \mathrm{H}_{15} \mathrm{NO}_{3}$ & 90 & $179-180$ \\
\hline 11 & , & $m-\mathrm{NO}_{2}$ & $\mathrm{C}_{21} \mathrm{H}_{15} \mathrm{NO}_{3}$ & 94 & $183-184\left(183-184^{19 b}\right)$ \\
\hline $1 \mathrm{~m}$ & $"$ & $p-\mathrm{NO}_{2}$ & $\mathrm{C}_{21} \mathrm{H}_{15} \mathrm{NO}_{3}$ & 96 & $189-190\left(190-191^{19 b}\right)$ \\
\hline $2 \mathrm{a}$ & 9H-Fluorenyl & $\mathrm{H}$ & $\mathrm{C}_{22} \mathrm{H}_{16} \mathrm{O}$ & 95 & $149-150\left(150^{19 b}\right)$ \\
\hline $2 b$ & $"$ & $m-\mathrm{NH}_{2}$ & $\mathrm{C}_{22} \mathrm{H}_{17} \mathrm{NO}$ & 94 & $95-96$ \\
\hline $2 \mathrm{c}$ & , & $p-\mathrm{NH}_{2}$ & $\mathrm{C}_{22} \mathrm{H}_{17} \mathrm{NO}$ & 94 & $93-94$ \\
\hline $2 \mathrm{~d}$ & $"$ & $m-\mathrm{Br}$ & $\mathrm{C}_{22} \mathrm{H}_{15} \mathrm{BrO}$ & 95 & $127-128$ \\
\hline $2 \mathrm{e}$ & , & $m-\mathrm{Cl}$ & $\mathrm{C}_{22} \mathrm{H}_{15} \mathrm{ClO}$ & 95 & $43-44\left(43-44^{19 b}\right)$ \\
\hline $2 f$ & , & $p-\mathrm{Cl}$ & $\mathrm{C}_{22} \mathrm{H}_{15} \mathrm{ClO}$ & 96 & $83-84\left(84-85^{19 b}\right)$ \\
\hline $2 \mathrm{~g}$ & " & $p-\mathrm{N}\left(\mathrm{CH}_{3}\right)_{2}$ & $\mathrm{C}_{24} \mathrm{H}_{21} \mathrm{NO}$ & 95 & $89\left(88-89^{19 b}\right)$ \\
\hline $2 \mathrm{~h}$ & $"$ & $p-\mathrm{OH}$ & $\mathrm{C}_{22} \mathrm{H}_{16} \mathrm{O}_{2}$ & 94 & $91-92\left(93-94^{19 b}\right)$ \\
\hline $2 \mathrm{i}$ & , & $p-\mathrm{OCH}_{3}$ & $\mathrm{C}_{23} \mathrm{H}_{18} \mathrm{O}_{2}$ & 96 & $94-95\left(94-95^{19 b}\right)$ \\
\hline $2 \mathrm{j}$ & $"$ & $p-\mathrm{CH}_{3}$ & $\mathrm{C}_{23} \mathrm{H}_{18} \mathrm{O}$ & 96 & $103-104$ \\
\hline $2 \mathrm{k}$ & $”$ & $o-\mathrm{NO}_{2}$ & $\mathrm{C}_{22} \mathrm{H}_{15} \mathrm{NO}_{3}$ & 91 & $90-91$ \\
\hline 21 & $"$ & $m-\mathrm{NO}_{2}$ & $\mathrm{C}_{21} \mathrm{H}_{15} \mathrm{NO}_{3}$ & 96 & $77-78\left(77-78^{19 b}\right)$ \\
\hline $2 \mathrm{~m}$ & , & $p-\mathrm{NO}_{2}$ & $\mathrm{C}_{21} \mathrm{H}_{15} \mathrm{NO}_{3}$ & 96 & $85-86\left(85-86^{19 b}\right)$ \\
\hline
\end{tabular}


(2E)-1-(4-Biphenyl)-3-(3-aminophenyl)-2-propen-1-one (1b)

$\operatorname{IR}\left(\mathrm{KBr}, \mathrm{cm}^{-1}\right): v=1693(\mathrm{CO}$ s-cis $), 1688(\mathrm{CO}$ s-trans $), 1025(\mathrm{CH}=\mathrm{CH}), 3436\left(\mathrm{NH}_{2}\right) ;{ }^{1} \mathrm{H}$ $\operatorname{NMR}\left(\mathrm{CDCl}_{3}, \mathrm{ppm}\right): \delta=7.831(\mathrm{~d}, 1 \mathrm{H} \alpha), 7.910(\mathrm{~d}, 1 \mathrm{H} \beta), 6.341-7.824(\mathrm{~m}, 13 \mathrm{H} \mathrm{Ar}-\mathrm{H}), 3.986(\mathrm{~s}$, $\left.2 \mathrm{H}-\mathrm{NH}_{2}\right) ;{ }^{13} \mathrm{C} \mathrm{NMR}\left(\mathrm{CDCl}_{3}, \mathrm{ppm}\right): \delta=121.540\left(\mathrm{C}_{\alpha}\right) 143.067\left(\mathrm{C}_{\beta}\right), 188.601(\mathrm{CO}) ; \quad$ Anal. Calcd: C, 84.25; H, 5.72; N, 4.68; Found: C, 8.23; H, 5.69; N, 4.59\%.

(2E)-1-(4-Biphenyl)-3-(4-aminophenyl)-2-propen-1-one (1c)

$\mathrm{IR}\left(\mathrm{KBr}, \mathrm{cm}^{-1}\right): v=1666(\mathrm{CO}$ s-cis $), 1655(\mathrm{CO}$ s-trans $), 991(\mathrm{CH}=\mathrm{CH}), 3576\left(-\mathrm{NH}_{2}\right) ;{ }^{1} \mathrm{H}$ $\operatorname{NMR}\left(\mathrm{CDCl}_{3}, \mathrm{ppm}\right): \delta=8.146(\mathrm{~d}, 1 \mathrm{H} \alpha), 8.245(\mathrm{~d}, 1 \mathrm{H} \beta), 7.311-7.767(\mathrm{~m}, 13 \mathrm{H} \mathrm{Ar}-\mathrm{H}), 4.034(\mathrm{~s}$, $\left.2 \mathrm{H}-\mathrm{NH}_{2}\right) ;{ }^{13} \mathrm{C} \mathrm{NMR}\left(\mathrm{CDCl}_{3}, \mathrm{ppm}\right): \delta=117.810\left(\mathrm{C}_{\alpha}\right) 140.852\left(\mathrm{C}_{\beta}\right)$; Anal. Calcd: $\mathrm{C}, 84.25 ; \mathrm{H}$, 5.72; N, 4.68; Found: C, 84.21; H, 5.70; N, 4.84\%.

(2E)-1-(4-Biphenyl)-3-(3-bromophenyl)-2-propen-1-one (1d)

$\mathrm{IR}\left(\mathrm{KBr}, \mathrm{cm}^{-1}\right): v=1682(\mathrm{CO} s$-cis $), 1668$ (CO s-trans), $1015(\mathrm{CH}=\mathrm{CH}) ;{ }^{1} \mathrm{H} \mathrm{NMR}\left(\mathrm{CDCl}_{3}\right.$, $\mathrm{ppm}): \delta=8.017(\mathrm{~d}, 1 \mathrm{H} \alpha), 8.305(\mathrm{~d}, 1 \mathrm{H} \beta), 7.455-8.032(\mathrm{~m}, 13 \mathrm{H} \mathrm{Ar}-\mathrm{H}) ;{ }^{13} \mathrm{C} \mathrm{NMR}\left(\mathrm{CDCl}_{3}\right.$, ppm): $\delta=121.111\left(\mathrm{C}_{\alpha}\right) 142.367\left(\mathrm{C}_{\beta}\right), 191.667(\mathrm{CO})$; Anal. Calcd: C, 69.44; H, 4.16; Found: C, 69.39; H, 4.13\%.

(2E)-1-(4-Biphenyl)-3-(4-dimethylaminophenyl)-2-propen-1-one (1g)

$\operatorname{IR}\left(\mathrm{KBr}, \mathrm{cm}^{-1}\right): v=1662(\mathrm{CO}$ s-cis $), 1651(\mathrm{CO}$ s-trans $), 990(\mathrm{CH}=\mathrm{CH}), 3566\left(-\mathrm{NH}_{2}\right) ;{ }^{1} \mathrm{H}$ $\operatorname{NMR}\left(\mathrm{CDCl}_{3}, \mathrm{ppm}\right): \delta=8.102(\mathrm{~d}, 1 \mathrm{H} \alpha), 8.123(\mathrm{~d}, 1 \mathrm{H} \beta), 7.656-8.379(\mathrm{~m}, 13 \mathrm{H} \mathrm{Ar}-\mathrm{H}), 2.58(\mathrm{~s}$, $\left.6 \mathrm{H}-\left(\mathrm{CH}_{3}\right)_{2}\right) ;{ }^{13} \mathrm{C} \quad \mathrm{NMR}\left(\mathrm{CDCl}_{3}, \mathrm{ppm}\right): \delta=117.810\left(\mathrm{C}_{\alpha}\right)$ 141.333. $\left.\mathrm{C}_{\beta}\right), \quad 192.200(\mathrm{CO})$, 42.821 $\left(\mathrm{CH}_{3}\right)$; Anal. Calcd: C, 84.37; H, 6.46; N, 4.28; Found: C, 84.34; H, 6.40; N, $4.25 \%$.

(2E)-1-(4-Biphenyl)-3-(2-nitrophenyl)-2-propen-1-one ( $1 \mathrm{k})$

$\operatorname{IR}\left(\mathrm{KBr}, \mathrm{cm}^{-1}\right): v=1680(\mathrm{CO}$ s-cis $), 1663(\mathrm{CO}$ s-trans $), 998(\mathrm{CH}=\mathrm{CH}) ;{ }^{1} \mathrm{H} \mathrm{NMR}\left(\mathrm{CDCl}_{3}\right.$, ppm): $\delta=8.269(\mathrm{~d}, 1 \mathrm{H} \alpha), 8.461(\mathrm{~d}, 1 \mathrm{H} \beta), 7.481-7.842(\mathrm{~m}, 13 \mathrm{H} \mathrm{Ar}-\mathrm{H}) ;{ }^{13} \mathrm{C} \mathrm{NMR}\left(\mathrm{CDCl}_{3}\right.$, ppm): $\delta=126.061\left(\mathrm{C}_{\alpha}\right)$ 141.391 $\left(\mathrm{C}_{\beta}\right), 188.791(\mathrm{CO})$; Anal. Calcd: C, 76.59; H, 4.55; N, 25; Found: C,56.52, H, 4.49; N, 4.18\%.

(2E)-1-(9H-Fluorene-2-yl)-3-(3-aminophenyl)-2-propen-1-one (2b)

$\operatorname{IR}\left(\mathrm{KBr}, \mathrm{cm}^{-1}\right): v=1663(\mathrm{CO}$ s-cis $), 1658(\mathrm{CO}$ s-trans $), 1012(\mathrm{CH}=\mathrm{CH}), 3436\left(\mathrm{NH}_{2}\right) ;{ }^{1} \mathrm{H}$ $\operatorname{NMR}\left(\mathrm{CDCl}_{3}, \mathrm{ppm}\right): \delta=7.481(\mathrm{~d}, 1 \mathrm{H} \alpha), 7.741(\mathrm{~d}, 1 \mathrm{H} \beta), 6.731-7.238(\mathrm{~m}, 13 \mathrm{H} \mathrm{Ar}-\mathrm{H}), 3.7346(\mathrm{~s}$, $\left.2 \mathrm{H}-\mathrm{NH}_{2}\right) ;{ }^{13} \mathrm{C} \mathrm{NMR}\left(\mathrm{CDCl}_{3}, \mathrm{ppm}\right): \delta=119.520\left(\mathrm{C}_{\alpha}\right)$ 140.067( $\left.\mathrm{C}_{\beta}\right), 193.641(\mathrm{CO})$; Anal. Calcd: C, 84.37; H, 6.46; N, 4.28; Found: C, 84.33; H, 6.42; N, 4.19\%.

(2E)-1-(9H-Fluorene-2-yl)-3-(4-aminophenyl)-2-propen-1-one (2c)

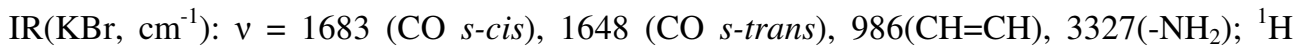
$\mathrm{NMR}\left(\mathrm{CDCl}_{3}, \mathrm{ppm}\right): \delta=8.124(\mathrm{~d}, 1 \mathrm{H} \alpha), 8.214(\mathrm{~d}, 1 \mathrm{H} \beta), 7.351-7.776(\mathrm{~m}, 13 \mathrm{H} \mathrm{Ar}-\mathrm{H}), 4.103(\mathrm{~s}$, $\left.2 \mathrm{H}-\mathrm{NH}_{2}\right) ;{ }^{13} \mathrm{C} \mathrm{NMR}\left(\mathrm{CDCl}_{3}, \mathrm{ppm}\right): \delta=119.810\left(\mathrm{C}_{\alpha}\right) 141.805\left(\mathrm{C}_{\beta}\right), 188.925(\mathrm{CO})$; Anal. Calcd: C, 84.37; H, 6.46; N, 4.28; Found: C, 84.29; H, 6.52; N 4.25\%.

(2E)-1-(9H-Fluorene-2-yl)-3-(3-bromophenyl)-2-propen-1-one (2d)

$\operatorname{IR}\left(\mathrm{KBr}, \mathrm{cm}^{-1}\right): v=1673(\mathrm{CO}$ s-cis $), 1668(\mathrm{CO}$ s-trans $), 992(\mathrm{CH}=\mathrm{CH}) ;{ }^{1} \mathrm{H} \mathrm{NMR}\left(\mathrm{CDCl}_{3}\right.$, ppm): $\delta=8.101(\mathrm{~d}, 1 \mathrm{H} \alpha), 8.336(\mathrm{~d}, 1 \mathrm{H} \beta), 7.723-8.01(\mathrm{~m}, 13 \mathrm{H} \mathrm{Ar}-\mathrm{H}) ;{ }^{13} \mathrm{C} \mathrm{NMR}\left(\mathrm{CDCl}_{3}, \mathrm{ppm}\right)$ : $\delta=122.123\left(\mathrm{C}_{\alpha}\right)$ 139.316( $\left.\mathrm{C}_{\beta}\right), 191.667(\mathrm{CO})$; Anal. Calcd: C, 67.36; H, 4.62; Found: $\mathrm{C}$, $67.34 ; \mathrm{H}, 4.59 \%$. 
(2E)-1-(9H-Fluorene-2-yl)-3-(3-dimethylaminophenyl)-2-propen-1-one (2g)

$\operatorname{IR}\left(\mathrm{KBr}, \mathrm{cm}^{-1}\right): v=1697(\mathrm{CO}$ s-cis $), 1654(\mathrm{CO}$ s-trans $), 978(\mathrm{CH}=\mathrm{CH}) ;{ }^{1} \mathrm{H} \mathrm{NMR}\left(\mathrm{CDCl}_{3}\right.$, ppm): $\delta=7.601(\mathrm{~d}, 1 \mathrm{H} \alpha), 7.862(\mathrm{~d}, 1 \mathrm{H} \beta), 6.765-7.510(\mathrm{~m}, 13 \mathrm{H} \mathrm{Ar}-\mathrm{H}), 2.648\left(\mathrm{~s}, 6 \mathrm{H}-\left(\mathrm{CH}_{3}\right)_{2}\right)$; ${ }^{13} \mathrm{C} \mathrm{NMR}\left(\mathrm{CDCl}_{3}, \mathrm{ppm}\right): \delta=119.880\left(\mathrm{C}_{\alpha}\right)$ 139.813. $\left.\mathrm{C}_{\beta}\right), 196.235(\mathrm{CO}), 43.170\left(\mathrm{CH}_{3}\right)$; Anal. Calcd: C, 81.12; H, 5.91; N, 3.90; Found: C, 81.05; H, 5.68; N, 3.88\%.

(2E)-1-(9H-Fluorene-2-yl)-3-(3-methylphenyl)-2-propen-1-one (2j)

$\operatorname{IR}\left(\mathrm{KBr}, \mathrm{cm}^{-1}\right): v=1668(\mathrm{CO}$ s-cis $), 1672(\mathrm{CO} s$-trans $), 1015(\mathrm{CH}=\mathrm{CH}) ;{ }^{1} \mathrm{H} \mathrm{NMR}\left(\mathrm{CDCl}_{3}\right.$, ppm): $\delta=7.928(\mathrm{~d}, 1 \mathrm{H} \alpha), 8.394(\mathrm{~d}, 1 \mathrm{H} \beta), 7.331-7.914(\mathrm{~m}, 13 \mathrm{H} \mathrm{Ar}-\mathrm{H}) 2.324\left(\mathrm{~s}, 3 \mathrm{H}-\mathrm{CH}_{3}\right) ;{ }^{13} \mathrm{C}$ $\mathrm{NMR}\left(\mathrm{CDCl}_{3}, \mathrm{ppm}\right): \delta=122.834\left(\mathrm{C}_{\alpha}\right) 144.621\left(\mathrm{C}_{\beta}\right), 198.294(\mathrm{CO}), 25.521\left(-\mathrm{CH}_{3}\right)$; Anal. Calcd: C, 89.00; H, 5.80; Found: C, 88.97; H, 5.81\%.

(2E)-1-(9H-Fluorene-2-yl)-3-(2-nitrophenyl)-2-propen-1-one (2k)

$\operatorname{IR}\left(\mathrm{KBr}, \mathrm{cm}^{-1}\right): v=1698(\mathrm{CO}$ s-cis $), 1678$ (CO s-trans), 974(CH=CH); ${ }^{1} \mathrm{H} \mathrm{NMR}\left(\mathrm{CDCl}_{3}\right.$, ppm): $\delta=7.829(\mathrm{~d}, 1 \mathrm{H} \alpha), 8.516(\mathrm{~d}, 1 \mathrm{H} \beta), 7.481-7.784(\mathrm{~m}, 13 \mathrm{H} \mathrm{Ar}-\mathrm{H}) ;{ }^{13} \mathrm{C} \mathrm{NMR}\left(\mathrm{CDCl}_{3}\right.$, ppm): $\delta=126.061\left(\mathrm{C}_{\alpha}\right)$ 141.391( $\left.\mathrm{C}_{\beta}\right), 196.253(\mathrm{CO})$; Anal. Calcd: C, 77.41; H, 4.43; N, 4.10; Found: C, 77.56; H, 4.39; N, 4.07\%.

The catalyst was recycled by washing the solid reagent remained on the filter by ethyl acetate $(20 \mathrm{ml})$ followed by drying in an oven at $50^{\circ} \mathrm{C}$ for $2 \mathrm{~h}$.and it can be reusable for another reaction run.

\section{Conclusion}

This method is a very efficient and selective protocol for crossed-aldol condensation of ketones and aldehydes to produced high yield in the presence of a reusable and environmentally beginning catalyst silica-sulphuric acid. Operative simplicity, easy workup procedure, better yield including washing the mixture followed by evaporation of the solvent is another advantage of this method.

\section{Acknowledgement}

The authors thankful to Prof. Dr. P. Ananthakrishana Nadar, Secretary, International Group of Correlation Analysis in Chemistry, Currently Emeritus Scientist, Faculty of Chemical Sciences, Bharath Institute of Technology, (Deemed University), Chennai for NMR data analysis.

\section{References}

1 (a) Wade L G, Organic Chemistry, $6^{\text {th }}$ Ed, Printice Hall. Upper Saddle River, New Jersey, 2005; 1056-1066, ISBN 013187151. (b). Smith M B and March J, Advanced Organic Chemistry, $5^{\text {th }}$ ed, Wiley Interwcience, New York, 2001; 1218-1223, ISBN 0-471-58589-0. (c). Mahrwald R, Modern Aldol reactions, Volumes 1 and 2, WileyVCH Verlag GmbH \& Co. KGaA. Weinheim, Germany, 2004, ISBN 3-527-30714-1.

2 (a) Heathcock C H Org. Syn. 1991, 2, 133. (b) Mukaiyama T Org. React. 1983, 28, 203; (c) Perterson J Chem Ind. 1988, 12, 390.

3 (a) Saini R K, Choudhary A S, Joshi Y C and Joshi P E. J. Chem. 2005, 2(9). (b) Dhar D N, Chemistry of Chalcones and Related Compounds; Wiley: N. Y., 1981.

4 Wu J H, Wang X H, Yi Y H and Lee K H Bioorg. \& Med. Chem. Lett.2003, 13, 1813.

5 (a) Nam N H, Kim Y, You Y J, Hong D H, Kim H M and Ahn B Z Eur. J. Med. Chem. 2003, 38, 179. (b) Saydam G, Aydin H H, Sahin F, Kucukoglu O, Erciyas E, Terzioglu E, Buyukkececi F and Omay S B Leukemia Res. 2003, 27, 57. 
6 (a) Wu X, Wilairat P and Go M L Bioorg. \& Med. Chem. Lett. 2002, 12, 2299. (b) Dominguez J N, Charris J. E, Lobo G, Dominguez N G, Moreno M M, Riggione F, Sanchez E, Olson J and Rosenthal P J Eur. J. Med. Chem. 2001, 36, 555.

(a) Tuchinda P, Reutrakul V, Claeson P, Pongprayoon U, Sematong T, Santisuk T, and Taylor W C Phytochem. 2002, 59,169. (b) Herencia F, Ferrandiz M L, Ubeda A, Dominguez J N, Charris J E, Lobo G M and Alcaraz M J Bioorg. \& Med. Chem. Lett. 1998, 8,1169.

8 (a). Xia Y, Yang Z Y, Xia P, Bastow K F, Nakanishi Y and Lee K H Bioorg. \& Med. Chem. Lett. 2000, 10, 699. (b) Ducki S, Forrest R, Hadfield J A, Kendall A, Lawrence N J, McGown A T and Rennison D Bioorg. \& Med. Chem. Lett. 1998, 8, 1051 .

9 Mears J R, Sailes H E, Watts J P and Whiting A Arkivoc, 2006, 1, 95.

10 (a) Guthrie W Can J. Chem. 1991, 69, 339. (b) Guthrie W, J. Am. Chem. Soc. 1991, 109, 6609. (c) Nielson A.T, Houlihan W J, Org React. 1968, 16, 1.

11 Hathaway B A J. Chem. Educ. 1987, 64, 367.

12 Nakano T, Irifune S, Umano S, Inada A, Ishii Y and Ogawa M J. Org. Chem. 1987, 52, 2239.

13 Irie K and Watanabe K Bull. Chem. Soc. Jpn. 1980, 53, 1366.

14 Wang L, Shao J and Zhong Q Synth. Communn. 1987, 27351.

15 Yadav J S, Subba Reddy B V and Sarma JARP Synth. Communn. 2002, 32, 893.

16 Iranpoor N, Zeynizadeli B and Aghapour A Chem. Res. (S) 1999, 554.

17 (a) Salehi P, Khodaei M M, Zolfigol M A and Keyvan A Monatsh. Chem. 2002, 133, 1291. (b) Deng G S and Ren T G Synth. Communn. 2003, 33, 2995.

18 Kalluraya B and Ray G Indian J. Chem. 2003, 2(B), 2556.

19 (a) Sung D D and Ananthakrishna Nadar P Indian J. Chem. 2000, 39(A), 1066. (b) Sung D D and Ananthakrishna Nadar P Bull. Korean Chem. Soc. 1999, 20(12), 1487. 20 Basaif S A, Sobahi T R, Khalil A K and Hassan M A Bull. Korean Chem. Soc. 2005, 26, 1677.

21 Riego J M, Sedin Z, Zaldivar J M, Marziano N C and Tortato C Tertahedran Lett. 1966, 43, 54.

22 Turro N J Tetrahedran. 1987, 43, 1589.

23 Thirunarayanan G and Vanangamudi G Arkivoc, 2006, 12, 58.

24 (a) Zolfigoal M.A, Torabi M and Mallkapour S E Tetrahedran. 2001, 57. 8381. (b). Shirini F, Zolfigoal M A, Mallakpour B, Mallakapour S E, Hajipour A R and Baltork I M Tetrahedran Lett. 2002, 43, 1555.

25 (a) Zolfigol M A Tertahedron. 2001, 37, 9509. (b) Mirjalili B F, Zolfigol M A and Bamoniri A J. Korean Chem. Soc. 2001, 45, 546. (c) Zolfigol M A and Bamoniri A Synlett. 2002, 1621. (d) Mirjalili B F, Zolfigol M A and Bamoniri A Molecules. 2002, 7, 751. (e) Zolfigol M A, Shirini F, Ghobani-Choghamarani A and Mohamadpoor-Baltork I Green Chem. 2002, 4, 562. (f) Zolfigol M A, Chelardoli G A and Mallakpour S E Synth. Commun. 2003, 33, 833. 


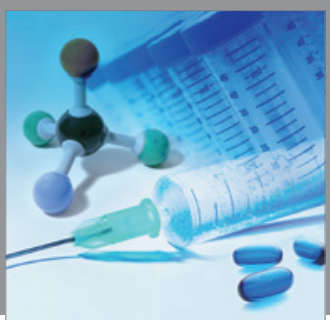

International Journal of

Medicinal Chemistry

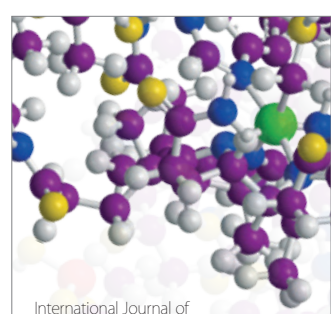

Carbohydrate Chemistry

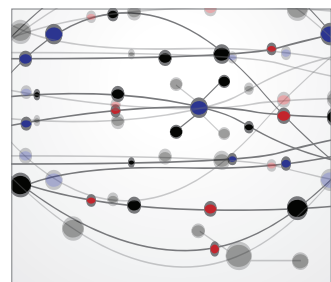

The Scientific World Journal
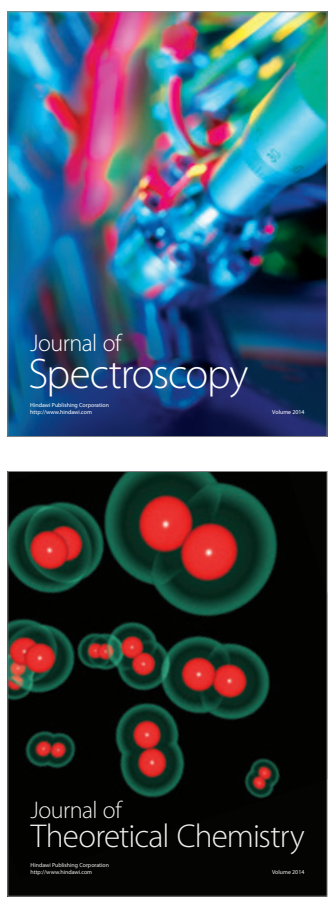
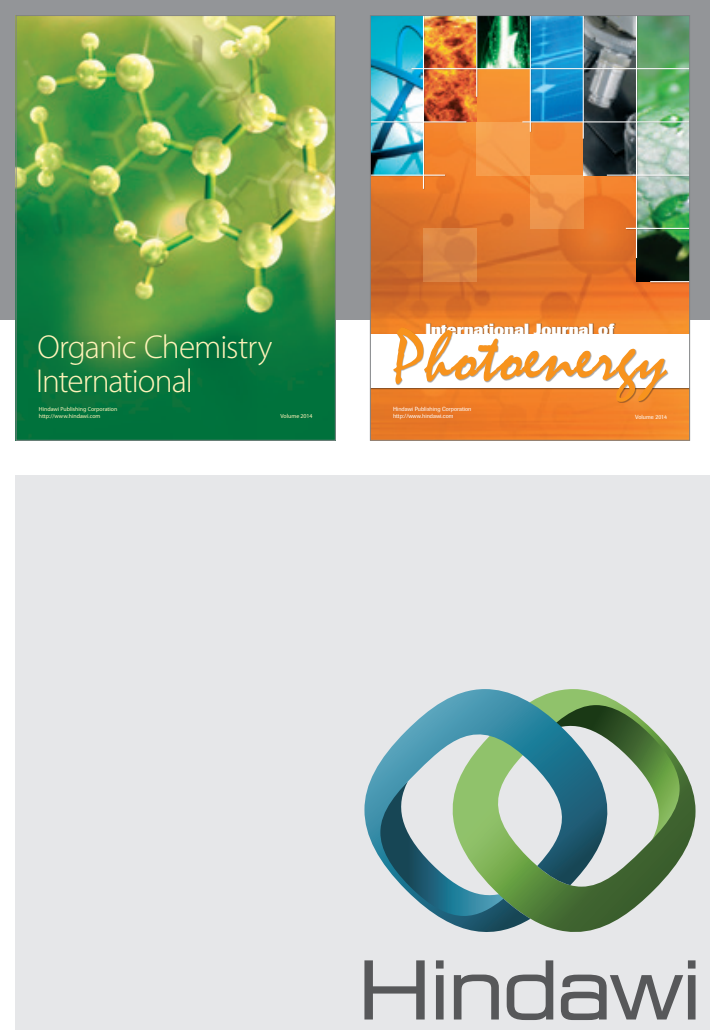

Submit your manuscripts at

http://www.hindawi.com
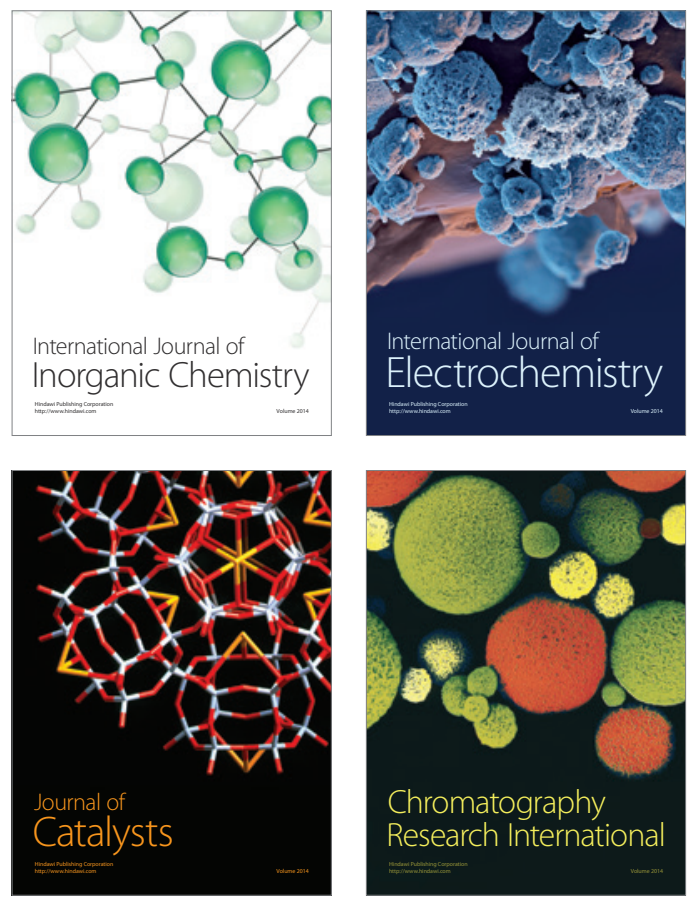
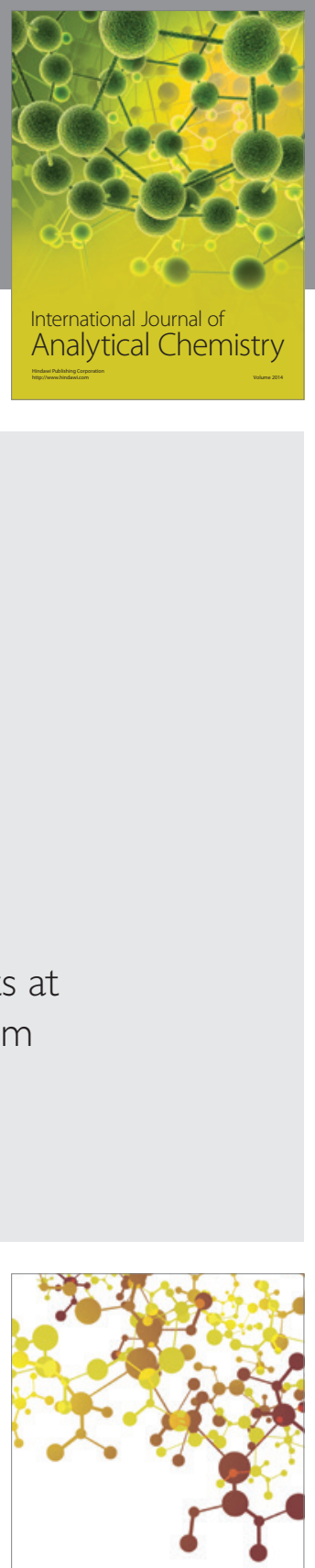

Journal of

Applied Chemistry
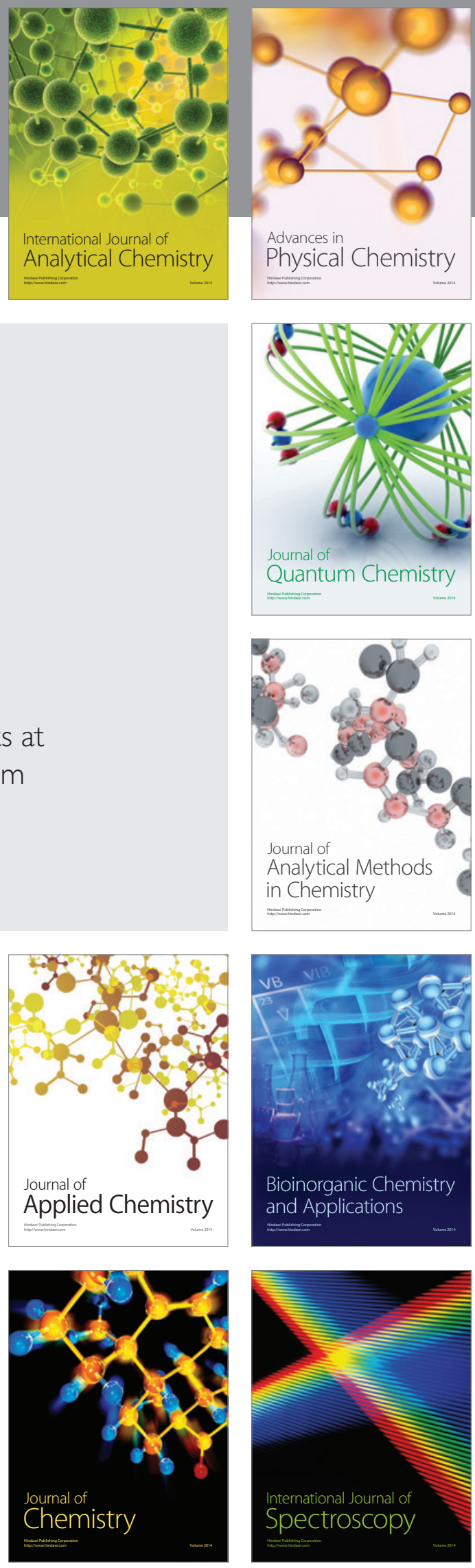